\title{
Effects of a 12-week workplace counseling program on physical activity and low back pain: a pilot randomized controlled study
}

Kazuhiro Shimo ( $\nabla$ shimo@reha.kobegakuin.ac.jp )

Kobe Gakuin University https://orcid.org/0000-0002-8575-6186

Mami Hasegawa

Aichi Medical University

Seiko Mizutani

Nihon Fukushi University

Tomomi Hasegawa

Aichi Medical University

Takahiro Ushida

Aichi Medical University

Research article

Keywords: workplace intervention, physical activity counseling, physical activity, low back pain, workers

Posted Date: April 14th, 2020

DOI: https://doi.org/10.21203/rs.2.20881/v2

License: (9) This work is licensed under a Creative Commons Attribution 4.0 International License. Read Full License

Version of Record: A version of this preprint was published at Journal of Back and Musculoskeletal Rehabilitation on April 28th, 2021. See the published version at https://doi.org/10.3233/BMR-200178. 


\section{Abstract}

Background: Physical activity (PA) is essential in the management and rehabilitation of low back pain (LBP). However, it is not clear that PA interventions in the workplace can improve LBP. This study aimed to investigate the effects of workplace counseling on PA levels, and LBP and physical function among workers.

Methods: We recruited 37 people with 12 weeks of LBP who worked as office staff or machinery mechanic in a manufacturing company in Aichi, Japan. Participants were randomly assigned to the intervention group $(n=20)$ or control group $(n=17)$. All participants of both groups were affixed with waist-worn accelerometers to monitor PA. The intervention group also received a program of face-to-face counseling with a physical therapist or nurse once a week for 12 weeks to reassure and encourage participants to maintain a high level of PA. PA, LBP severity and physical function were assessed at baseline, 3 and 6 months.

Results: Baseline characteristics were similar in both groups. PA was significantly higher in the intervention group than in the control group at 3 and 6 months. In the intervention group, PA and physical function significantly increased at 3 and 6 months from baseline, and LBP severity at 6 months improved significantly from baseline. We calculated the effect size of the PA workplace counseling and found that it had a medium-to-large effect on PA, LBP severity and physical function.

Conclusions: Our data suggest that workplace PA intervention can increase PA and improve LBP among workers.

Trial registration: UMIN-CTR Clinical Trial UMIN000038864 (https://upload.umin.ac.jp/cgi-openbin/ctr_e/ctr_view.cgi?recptno=R000044321). Registered 12 December 2019, retrospectively registered.

\section{Background}

Chronic low back pain (LBP) has a high prevalence and significant social impact.[1] In Japan, the lifetime prevalence of LBP is estimated at $83 \%$, with nearly $10 \%$ of the population suffering from disabling LBP involving a workplace absence of $\geq 4$ consecutive days.[2] The annual economic cost of chronic pain due to the lost opportunity to work is estimated at US\$19.9 billion in Japan, where LBP is the most common type of chronic pain.[3] The prevalence of chronic musculoskeletal pain occurring in the low back is highest in people in their 30s to 50s, commonly known as the working generation.[4] However, more than half of the people with chronic musculoskeletal pain do not receive any form of treatment.[4] It seems apparent that many workers are suffering from LBP and would benefit from intervention.

Physical activity (PA) is an essential component of the management and rehabilitation of LBP.[5-8] Workplace health promotion programs have been shown to increase the PA of workers.[9] Previous studies have suggested that monitoring PA with an accelerometer, and the provision of feedback and specific advice on the benefits of walking, are two of many effective approaches.[10,11] Moreover, 
counseling based on the motivational interviewing model can improve exercise adherence and enhance PA levels.[12] Workplace PA interventions also reduce general musculoskeletal pain, as well as neck and shoulder pain,[13] but workplace interventions have a low effect on back pain according to a systematic review.[13] A major advantage of workplace interventions is that they provide easy access to workers; interventions that could improve LBP in workers might also have the potential to reduce social and economic losses.

The purpose of the study was to investigate the efficacy of workplace counseling on PA to reduce LBP. Therefore, we assessed whether a 12-week PA counseling program delivered by the authors (a physical therapist and two nurses) in a workplace setting would increase the levels of PA in workers and lead to improved LBP symptoms.

\section{Methods}

\section{Methods}

\section{Study design and participants}

This was pilot randomized controlled study conducted at a single manufacturing company in Aichi, Japan ( $n=471 ; 405$ men, 86\%). Eligible participants were full-time Japanese office workers and machinery mechanics aged 18 years or older with LBP symptoms for at least 12 weeks. Exclusion criteria included any cardiac, systemic or inflammatory diseases. Workers completed a self-administered, structured questionnaire for screening. We invited 131 workers who met the criteria to join the study, of which $29.8 \%(n=39)$ enrolled. Participants were assigned to the intervention group or control group by a randomized block design. Subjects were divided into five blocks (departments in the company) in order to avoid any bias toward the kind of work performed. A lottery method of manual randomization was conducted due to the small number of blocks and participants. To ensure allocation concealment, the randomization was carried out by a nurse who was not otherwise involved in the study. The intervention was provided free of charge to all participants.

This study was approved by the ethical review board of Aichi Medical University, and all participants provided written informed consent.

This study was funded by the Japanese Society of Physical Therapy.

\section{Intervention}

A total of 37 workers participated in this study and were assigned either to the intervention group $(n=20)$ or the control group $(n=17)$. We explained the potential relationship between PA and LBP and taught a series of exercises to be performed at home to all participants in both the intervention control groups in a single 60-min session. The home exercise program comprised seven floor stretches, four standing stretches to be held for $20 \mathrm{sec}$ on each side, three core stability exercises of 10 reps each, and $10 \mathrm{~min}$ of walking at a moderate speed. We instructed participants to engage in the program at least 5 days/week 
and then to gradually increase the number of steps and walking speed; we provided handouts with written instructions supplemented with diagrams to improve their understanding of the program components. All participants received a loaner waist-worn accelerometer (Lifecorder GS, Suzuken Co., Ltd., Japan) to monitor their PA, and were instructed to wear the accelerometer throughout the day except during sleep and water-related activities (e.g., bathing, swimming). Data were retrieved with PA analysis software (Lifelizer Coach, Suzuken Co., Ltd., Japan) every week to generate PA reports for each participant.

Participants in the intervention group received a workplace counseling program that aimed to increase the amount of daily PA with adequate pacing. The workplace counseling program was led by K.S., a physical therapist with 6 years of clinical experience at an interdisciplinary pain center, and two occupational health nurses with 6 and 22 years of career experience (M.H. and S.M.), respectively. Counseling sessions were carried out using a motivational interviewing approach, which had been routinely used in clinical practice by each of the program leaders. The program leaders visited the workplace and provided a 15-min face-to-face individual counseling session to each participant during working hours, once a week for 12 weeks. Each week the participants were given their PA results from the previous week and asked set a target PA for the next week, based on the report generated by the accelerometer and PA analysis software. We also monitored adherence to the home exercise program, provided guidance on how to perform the exercises, or suggested new exercises whenever necessary. Moreover, we reassured the participants about their progress, and encouraged them to maintain a high level of PA.

The control group participants were given PA reports delivered through their company's nurse, without counseling, once a week for 12 weeks.

\section{Outcomes}

Assessments were performed for all participants 2-4 weeks before their first scheduled intervention (baseline), and 1-3 weeks following the last day of the intervention (3-month time point). Follow-up assessments were conducted 6 months after the first scheduled intervention (6-month time point). Figure 1 shows a flow diagram of the study design.

\section{Primary outcome}

\section{Physical activity}

PA was measured by a waist-worn accelerometer, which assessed the average daily number of steps and motor activity (kcal) on a weekly basis.

\section{Secondary outcome}

\section{LBP severity}


LBP severity was assessed using a visual analog scale (VAS) scoring of maximum low back-specific pain during the last week. The participants were instructed to indicate, using an arrow, the intensity of pain on a $100 \mathrm{~mm}$ line, with 100 representing the worst pain and zero representing no pain. LBP-related disabilities on lifestyle were assessed using the Roland-Morris Disability Questionnaire (RDQ), which is a self-administered disability measure on a 24-point scale; greater levels of disability are reflected by higher numbers.[14] The RDQ was conducted using Japanese translations.[15]

\section{Physical function}

The physical function assessment measured endurance (6-min walking distance [6MD]) and flexibility (seated forward bends). For the 6MD, participants walked as far as possible on a flat, 50-m course, and the distance walked in 6 min was measured.[16] Seated forward bends were analyzed by measuring the distance from the fingertips to the toes with the knees fully extended using a seated forward-bend measurement instrument (Takei Scientific Instruments, Japan). Results were expressed as positive numbers when the fingertips passed the toes, and as negative numbers when the fingertips did not reach the toes.

\section{Statistical analysis}

Statistical analyses were performed using the Predictive Analytics Software, version 23 (IBM SPSS Statistics; SPSS Inc, Chicago, IL, USA). Demographic data are expressed as means and standard deviations (SD). Median and range were calculated for all parameters without demographic data; our data did not meet the requirements for a parametric test because of the non-normal distribution. Data at the baseline, 3 -month and 6 -month time points were first analyzed by the Friedman test $(p<0.05)$. A post hoc Wilcoxon signed-rank test procedure was performed to identify significant differences between the baseline, 3-month and 6-month time points $(p<0.025)$. Differences between the intervention and control groups at each time point were evaluated using the Mann-Whitney $U$ test $(p<0.016)$. We considered a $p$ value of $<0.05$ to be significant. However, for multiple outcome measures, the Bonferroni-adjusted significance level was calculated to account for the increased possibility of a type-1 error $(a=0.05)$. We also used " $r$ " as calculated by $Z$ translation to evaluate the magnitude of the effect size $(r=Z / \sqrt{N})$ according to Cohen,[17] where an $r$ value from 0.1 to $0.3=$ small effect size, 0.3 to $0.5=$ medium effect size, and $r>0.5=$ large effect size.

\section{Results}

The baseline demographic and clinical characteristics of the participants are shown in Table 1. There were no significant differences between the intervention and control groups in any of these characteristics. The study involved only male participants because none of the female invitees chose to enroll. Two enrolled participants, both assigned to the control group, declined to participate. No one in either group dropped out of the trial.

Table 1. Baseline demographics and clinical characteristics of the two study groups 


\begin{tabular}{|c|c|c|c|}
\hline \multirow[t]{2}{*}{ Parameters } & $\begin{array}{l}\text { Intervention group } \\
\qquad(\mathrm{n}=20)\end{array}$ & $\begin{array}{l}\text { Control group } \\
\qquad(\mathrm{n}=17)\end{array}$ & \multirow[t]{2}{*}{$\begin{array}{c}\mathrm{p} \text { value for difference between } \\
\text { groups }\end{array}$} \\
\hline & \multicolumn{2}{|c|}{ Mean (SD) or Median [Range] } & \\
\hline Age & $47.8(12.8)$ & $41.4(11.9)$ & 0.12 \\
\hline Gender: men (\%) & 100 & 100 & 1 \\
\hline $\operatorname{BMI}\left(\mathrm{kg} / \mathrm{m}^{2}\right)$ & $24.11(3.34)$ & $23.18(2.85)$ & 0.37 \\
\hline \multicolumn{4}{|l|}{ Physical activity } \\
\hline \multirow[t]{2}{*}{ Steps per day (no.) } & $10475(3060)$ & 10063 (3729) & \\
\hline & $\begin{array}{c}9879[6091- \\
16815]\end{array}$ & $\begin{array}{c}9465[5081- \\
18769]\end{array}$ & 0.577 \\
\hline \multirow{2}{*}{$\begin{array}{l}\text { Motor activity per day } \\
\text { (kcal) }\end{array}$} & $316.5(98.2)$ & $304.5(130.2)$ & \\
\hline & $\begin{array}{c}281.9[194.6- \\
489.3]\end{array}$ & $240[150.3-593.7]$ & 0.407 \\
\hline \multicolumn{4}{|l|}{ Low back pain severity } \\
\hline \multirow[t]{2}{*}{ VAS } & $38.1(24.9)$ & $33.1(21.6)$ & \\
\hline & $37[0-81]$ & $30[0-71]$ & 0.619 \\
\hline \multirow[t]{2}{*}{ RDQ } & $4.3(4.2)$ & $2.3(3.2)$ & \\
\hline & $3[0-15]$ & $1[0-10]$ & 0.74 \\
\hline \multicolumn{4}{|l|}{ Physical function } \\
\hline 6-min walking distance & $678.4(85.1)$ & $622.8(86.8)$ & \\
\hline$(\mathrm{m})$ & $694.5[455-816]$ & $635[442-770]$ & 0.026 \\
\hline \multirow[t]{2}{*}{$\begin{array}{l}\text { Seated forward bends } \\
\text { (cm) }\end{array}$} & $0.85(11.4)$ & $-1.9(12.4)$ & \\
\hline & $3.75[-19.5-18.5]$ & $2[-24.5-15]$ & 0.46 \\
\hline
\end{tabular}

Abbreviations: BMI, body mass index, VAS, visual analog scale; RDQ, Roland-Morris Disability Questionnaire.

Table 2 shows the outcomes at each time point after intervention. The effect size of workpalce PA intervention is presented in Table 3.

Table 2 Outcomes at each time point after intervention 


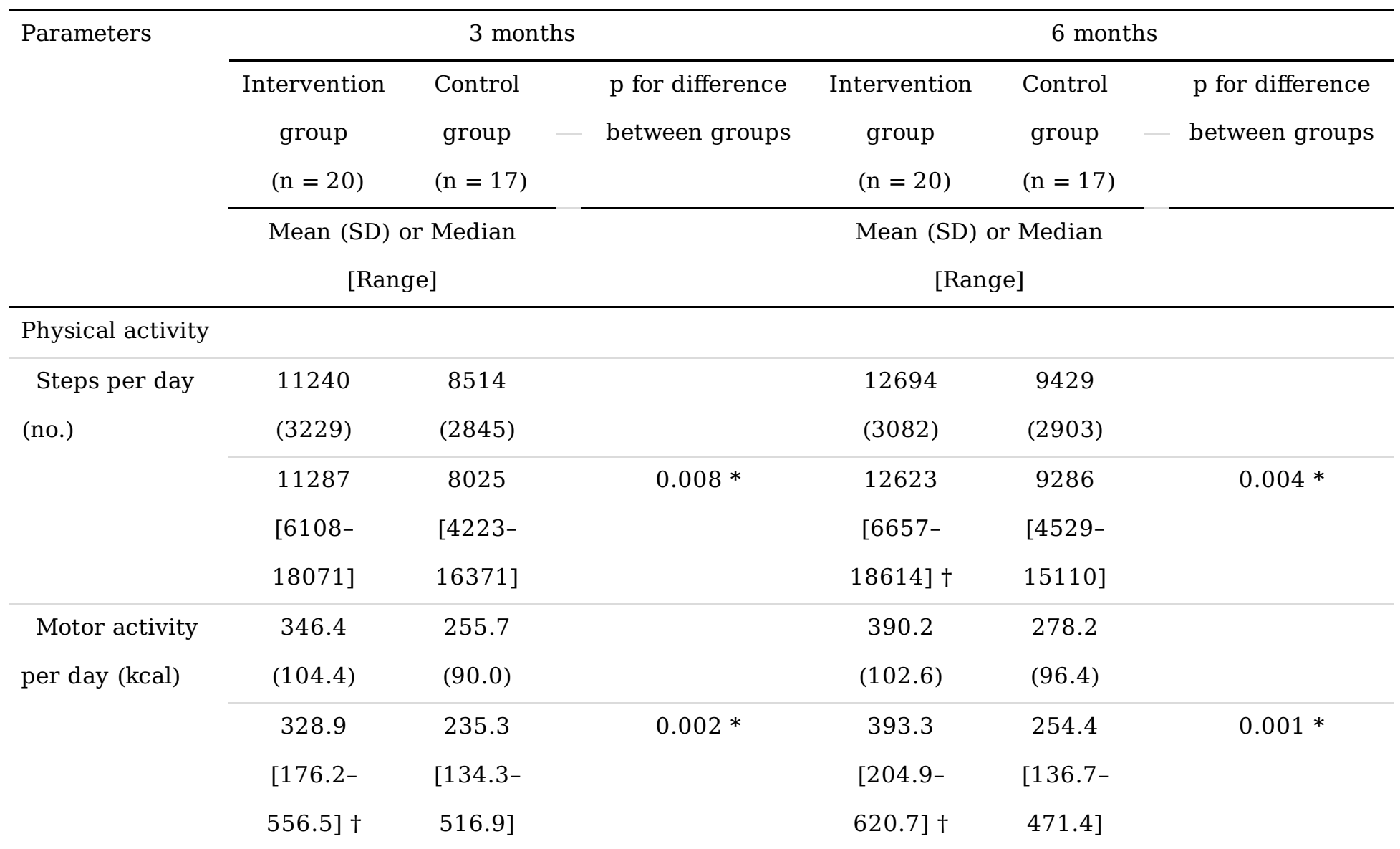

Low back pain

severity

\begin{tabular}{ccccccc} 
VAS & $29.2(25.9)$ & $36(26.0)$ & & $22.6(24.7)$ & $20.5(15.2)$ & \\
\cline { 2 - 7 } & $20[0-72]$ & $31[0-85]$ & 0.297 & $13[0-69] \dagger$ & $22[0-50]$ & 0.729 \\
\hline \multirow{2}{*}{ RDQ } & $2.6(2.5)$ & $2.7(3.5)$ & & $1.4(2.3)$ & $1.8(3.2)$ & \\
\hline
\end{tabular}

Physical

function

\begin{tabular}{|c|c|c|c|c|c|c|}
\hline \multirow{4}{*}{$\begin{array}{l}6 \text { min walking } \\
\text { distance }(\mathrm{m})\end{array}$} & 734.5 & 686.6 & & $743.7(83.0)$ & 695.5 & \\
\hline & $(67.8)$ & (68.3) & & & (64.9) & \\
\hline & 730 [617- & $685[600-$ & \multirow[t]{2}{*}{0.048} & 731 [620- & 700 [600- & 0.104 \\
\hline & $885] \dagger$ & $830] \dagger$ & & $1000] \dagger$ & $815] \dagger$ & \\
\hline \multirow{4}{*}{$\begin{array}{l}\text { Seated forward } \\
\text { bends }(\mathrm{cm})\end{array}$} & $5.4(9.1)$ & -1.3 & & $5(8.3)$ & $0.1(10.8)$ & \\
\hline & & 810.9) & & & & \\
\hline & $6[-12.5-$ & $-0.1[-23-$ & \multirow[t]{2}{*}{0.065} & $7[-10.5-$ & $-0.5[-22-$ & \multirow[t]{2}{*}{0.141} \\
\hline & 19.5] † & $15.5]$ & & $18] \dagger$ & 19] & \\
\hline
\end{tabular}

Notes: $* \mathrm{p}<0.016$ vs control. $\dagger p<0.025$ vs baseline in each group

Abbreviations: VAS, visual analog scale; RDQ, the Roland-Morris Disability Questionnaire. 
Table 3. Effect size

Workplace PA program intervention group

\begin{tabular}{|c|c|c|c|c|}
\hline \multirow[t]{4}{*}{ Parameters } & \multicolumn{4}{|c|}{$(n=20)$} \\
\hline & \multicolumn{2}{|c|}{ vs baseline } & \multicolumn{2}{|c|}{ vs control } \\
\hline & 3 months & 6 months & 3 months & 6 months \\
\hline & \multicolumn{4}{|c|}{ Effect size (r) } \\
\hline \multicolumn{5}{|l|}{ Physical activity } \\
\hline Steps per day & 0.34 & 0.54 & 0.43 & 0.47 \\
\hline Motor activity per day & 0.38 & 0.50 & 0.50 & 0.53 \\
\hline \multicolumn{5}{|l|}{ Low back pain severity } \\
\hline VAS & - & 0.51 & - & - \\
\hline $\mathrm{RDQ}$ & - & 0.50 & - & - \\
\hline \multicolumn{5}{|l|}{ Physical function } \\
\hline 6 min walking distance & 0.51 & 0.55 & - & - \\
\hline Seated forward bends & 0.56 & 0.43 & - & - \\
\hline
\end{tabular}

Abbreviations: PA, physical activity; VAS, visual analog scale; RDQ, Roland-Morris Disability Questionnaire.

\section{Physical activity}

As shown in Table 2, PA was significantly higher in the intervention group than in the control group at each time point, in terms of both the number of steps ( 3 and 6 months $p=0.008$ and 0.004 , respectively) and motor activity ( 3 and 6 months: $p=0.002$ and 0.001 ). The workplace intervention had a medium-tolarge effect (steps at 3,6 months: $r=0.43,0.47$; motor activity at 3,6 months: $r=0.50,0.53$ ) on PA (Table 3). In the intervention group, PA significantly improved at 3 and 6 months from baseline (steps at 6 months: $p=0.01$; motor activity at 3,6 months: $p=0.015,0.012)$, and showed a medium-to-large effect size (steps at 3,6 months: $r=0.34,0.54$; motor activity at 3,6 months: $r=0.38,0.50)($ Table 2,3$)$. By contrast, there was no significant change in PA from baseline in the control group (Table 2).

\section{LBP severity}

There were no significant differences in LBP severity between the groups for either the VAS or RDQ (Table 2). In the intervention group, LBP severity at 6 months improved significantly from baseline (VAS: $p=$ 0.001 ; RDQ: $p=0.002$ ) and showed a large effect size (VAS: $r=0.51 ; R D Q=0.50 ;$ Table 2, 3). There was no significant change in LBP severity from baseline in the control group (Table 2). 


\section{Physical function}

Physical function significantly improved from baseline at 3 and 6 months ( $6 \mathrm{MD}$ at 3,6 months: $\mathrm{p}=0.01$, 0.001 ; seated forward bends at 3,6 months: $p=0.001,0.007$; Table 2 ), with a medium-to-large effect size (steps at 3,6 months: $r=0.34,0.54$; motor activity at 3,6 months: $r=0.38,0.50$ ) (Table 3 ). There were also changes in 6MD in the control group, with significant improvements at 3 months $(p=0.001)$ and 6 months ( $p=0.001$ ) from baseline (Table 2 ). However, there were no significant changes in seated forward bends from baseline in control group (Table 2).

There were no adverse events in this study.

\section{Discussion}

A 12-week counseling program to increase PA among workers with LBP, administered in the workplace by a physical therapist or nurse, improved PA, LBP severity and physical function. Moreover, PA levels increased significantly in the intervention group compared with the control group. The effect size of improvement in outcomes was medium to large.

The PA of all of the study participants was monitored by an accelerometer and each received a weekly target PA. Only those in the intervention group, who received about $15 \mathrm{~min}$ of weekly face-to-face counseling, were motivated to increase their PA levels. These results are in agreement with a previous meta-analysis that showed that interventions delivered face-to-face to individuals are superior to other interventions to raise PA.[18] More specifically, the increased PA in our study was, at least in part, probably attributable to the regular reassurance and encouragement provided during the counseling sessions. At 6 months, there was a mean increase in PA of 2218.9 steps per day relative to baseline (95\% confidence interval [1254.1-3183.8]) in the intervention group, consistent with systematic reviews [19, 20] of research on pedometer-driven walking programs to increase PA. Furthermore, the systematic reviews $[19,20]$ report that this level of increase in PA improved musculoskeletal pain, physical function, body mass index, and systolic blood pressure. Workplace PA intervention has the potential to be applied for LBP as well as for the prevention and improvement of other health problem.

Several studies have shown that workplace PA intervention improves LBP. [21-23] Our study suggests that short-term PA counseling by a physical therapist or nurse delivered in the workplace can reduce LBP. In previous studies,[21-23] workplace intervention for LBP required 25-90 min of intervention per week. However, according to a systematic review,[13] these workplace PA interventions had a low effect size for the improvements in LBP (Hedges's $g=-0.21[-0.58,0.17])$. Our intervention comprised only about 15 min of counseling per week, which was shorter than that reported by most other studies. A shorter intervention time is theoretically advantageous because it reduces the time burden for both workers and employers, thereby encouraging persistence with the program and less dropout. Nevertheless, while there was a significant improvement in LBP severity in the intervention group compared with the baseline level, the difference in improvement between the intervention and control groups was not significant. We suspect that this result could be a reflection of the inclusion of participants with mild LBP, who might be 
more likely to experience improvements by simply wearing an accelerometer. In any case, it will be necessary to investigate contents of the intervention. Our intervention may have supported workers in managing their LBP more easily than previous interventions, but future studies will be challenged to determine the minimum frequency and amount of intervention required for maximal workplace PA improvement.

\section{Limitations}

There were several limitations to this study that warrant discussion. First, we had a small sample size and a single population. We conducted a randomized controlled study to reduce potential sources of bias, but did not secure multiple companies to participate. Furthermore, only about $30 \%$ of the 131 invited participants ultimately enrolled in the study. Although our workplace PA intervention had a moderate-tohigh effect size, clustered randomized trials with greater sample sizes are needed to evaluate and validate this intervention. Second, we included participants who were experiencing mild LBP lasting at least 12 weeks at the time of recruitment of the study. However, several participants were not suffering from LBP at the baseline time point. For these reasons, the generalizability of our findings is limited, and thus should be interpreted with caution. Third, we did not evaluate psychological factors. There is evidence that psychological factors play a significant role in LBP, and that exercise can positively influence such factors including fear-avoidance beliefs, catastrophic thinking and self-efficacy in people with chronic LBP. $[24,25]$ The mechanisms underlying the association between our intervention and improvement of LBP are unclear, and should be investigated in a future study on workplace PA intervention. Last, although we did check participants for adherence to the home exercise program, we did not measure adherence quantitatively. A future investigation of the minimum intervention for maximal workplace PA improvement should include a quantitative measurement of the home exercise component.

\section{Conclusion}

This 12-week study comprising a home exercise program together with PA counseling by a physical therapist or nurse, delivered as 15-min face-to-face sessions in the workplace, increased PA levels and improved LBP in workers. Workplace PA interventions have the potential to increase PA and improve LBP severity and physical function.

\section{List Of Abbreviations}

6MD: 6-min walking distance

LBP: Low back pain

PA: Physical activity

RDQ: Roland-Morris Disability Questionnaire 
SD: standard deviations

VAS: Visual analog scale

BMl: Body mass index

\section{Declarations}

\section{Ethics approval and consent to participate}

This study was approved by the ethical review board of Aichi Medical University (13-053), and all participants provided written informed consent.

\section{Consent for publication}

Not applicable.

\section{Availability of data and materials}

The datasets used and/or analyzed during the current study are available from the corresponding author on reasonable request.

\section{Competing interests}

The authors declare that they have no competing interests.

\section{Funding}

This study was funded by the Japanese Society of Physical Therapy (grant no A-11-15). The funding sources did not influence study design, collection, analysis and interpretation of data, writing, or the decision to submit the manuscript for publication.

\section{Authors' Contributions}

All authors made significant contributions to this manuscript and project in general. All authors read and approve this manuscript. We have outlined the specific contributions below: Contributions to conception and design of the study: All authors (KS, MH, SM, TH, TU). Drafted manuscript: KS. Critically reviewed and revised the manuscript: All authors (KS, MH, SM, TH, TU). Give final approval of manuscript version to be published: All authors (KS, MH, SM, TH, TU). Agreed to be accountable for all aspects of manuscript and trial work: All authors (KS, MH, SM, TH, TU).

\section{Acknowledgments}

The authors would like to thank Kaori Futatsuishi and Naoki Mizuno for their help in connection with research participants. The authors would like to thank Enago (www.enago.jp) for the English language 
review.

\section{Author's information}

Institute of Physical Fitness, Sports Medicine and Rehabilitation, Aichi Medical University, 1-1 Karimata, Yazako, Nagakute, Aichi, Japan

Kazuhiro Shimo, Tomomi Hasegawa, Takahiro Ushida

Multidisciplinary Pain Center, Aichi Medical University, 1-1 Karimata, Yazako, Nagakute, Aichi, Japan

Kazuhiro Shimo, Takahiro Ushida

Faculty of Rehabilitation, Kobe Gakuin University, 518 Arise, Ikawadani-cho, Nishi-ku, Kobe, Hyogo, Japan Kazuhiro Shimo

School of Nursing, Aichi Medical University, 1-1 Karimata, Yazako, Nagakute, Aichi, Japan

Mami Hasegawa, Seiko Mizutani

Faculty of Nursing, Nihon Fukushi University, 229 Kawaminami, Ota-machi, Toukai, Aichi, Japan

Seiko Mizutani

Corresponding author

Correspondence to Kazuhiro Shimo

\section{References}

1. GBD 2017 Disease and Injury Incidence and Prevalence Collaborators. Global, regional, and national incidence, prevalence, and years lived with disability for 354 diseases and injuries for 195 countries and territories, 1990-2017: a systematic analysis for the Global Burden of Disease Study 2017. Lancet 2018;392:1789-1858.

2. Fujii T, Matsudaira K. Prevalence of low back pain and factors associated with chronic disabling back pain in Japan. Eur Spine J. 2013;22:432-8.

3. Inoue S, Kobayashi F, Nishihara M, Arai YC, Ikemoto T, Kawai T, et al. Chronic pain in the Japanese community-prevalence, characteristics and impact on quality of life. PLoS One 2015;10:e0129262

4. Nakamura M, Nishiwaki Y, Ushida T, Toyama Y. Prevalence and characteristics of chronic musculoskeletal pain in Japan. J Orthop Sci. 2011;16:424-2.

5. Burton AK. How to prevent low back pain. Best Pract Res Clin Rheumatol. 2005;19:541-55.

6. Koes BW, van Tulder M, Lin CW, Macedo LG, McAuley J, Maher C. An updated overview of clinical guidelines for the management of non-specific low back pain in primary care. Eur Spine J. 
2010;19:2075-94.

7. Koes BW, van Tulder MW, Thomas S. Diagnosis and treatment of low back pain. BMJ 2006;332:1430-4.

8. van Tulder M, Becker A, Bekkering T, Breen A, del Real MT, Hutchinson A, et al. Chapter 3. European guidelines for the management of acute nonspecific low back pain in primary care. Eur Spine J. 2006;15:S169-91.

9. Ashe MC. Physical activity and workplace sedentary behaviour. Physiother Can. 2012;64:1-5.

10. Heath GW, Heath GW, Parra DC, Sarmiento OL, Andersen LB, Owen N, et al. Evidence-based intervention in physical activity: lessons from around the world. Lancet 2012;380:272-81.

11. Savigny P, Watson P, Underwood M; on behalf of the Guideline Development Group. Early management of persistent non-specific low back pain: summary of NICE guidance. BMJ 2009;338:b1805.

12. Stonerock GL, Blumenthal JA. Role of counseling to promote adherence in healthy lifestyle medicine: strategies to improve exercise adherence and enhance physical activity. Prog Cardiovasc Dis. 2017;59:455-62.

13. Moreira-Silva I, Teixeira PM, Santos R, Abreu S, Moreira C, Mota J. The Effects of workplace physical activity programs on musculoskeletal pain: a systematic review and meta-analysis. Workplace Health Saf. 2016;64:210-22.

14. Roland M, Morris R. A study of the natural history of back pain. Part I: development of a reliable and sensitive measure of disability in low-back pain. Spine 1983;8:141-4.

15. Suzukamo Y, Fukuhara S, Kikuchi S, Konno S, Roland M, Iwamoto Y, et al. Validation of the Japanese version of the Roland-Morris Disability Questionnaire. J Orthop Sci. 2003;8:543-8.

16. Laboratories ATSCoPSfCPF. ATS statement: guidelines for the six-minute walk test. Am J Respir Crit Care Med. 2002;166:111-7.

17. Cohen J. Statistical power analysis for the behavioral sciences. Lawrence Erlbaum Associates. Hillsdale, NJ. 1988.

18. Conn VS, Hafdahl AR, Mehr DR. Interventions to increase physical activity among healthy adults: meta-analysis of outcomes. Am J Public Health. 2011;101:751-8.

19. Bravata DM, Smith-Spangler C, Sundaram V, Gienger AL, Lin N, Lewis R, et al. Using pedometers to increase physical activity and improve health: a systematic review. JAMA 2007;298:2296-304.

20. Mansi S, Milosavljevic S, Baxter GD, Tumilty S, Hendrick P. A systematic review of studies using pedometers as an intervention for musculoskeletal diseases. BMC Musculoskelet Disord. 2014 ; 15 : 231.

21. Kellett KM, Kellett DA, Nordholm LA. Effects of an exercise program on sick leave due to back pain. Phys Ther. 1991;71:283-91.

22. Horneij E, Hemborg B, Jensen I, Ekdahl C. No significant differences between intervention programmes on neck, shoulder and low back pain: a prospective randomized study among home- 
care personnel. J Rehabil Med. 2001;33:170-6.

23. Sjogren T, Nissinen KJ, Jarvenpaa SK, Ojanen MT, Vanharanta H, Malkia EA. Effects of a workplace physical exercise intervention on the intensity of headache and neck and shoulder symptoms and upper extremity muscular strength of office workers: a cluster randomized controlled cross-over trial. Pain. 2005;116:119-28.

24. Smeets RJ, Vlaeyen JW, Kester AD, Knottnerus JA. Reduction of pain catastrophizing mediates the outcome of both physical and cognitive-behavioral treatment in chronic low back pain. J Pain. 2006;7:261-71.

25. Mannion AF, Junge A, Taimela S, Muntener M, Lorenzo K, Dvorak J. Active therapy for chronic low back pain: part 3 . Factors influencing self-rated disability and its change following therapy. Spine 2001;26:920-9.

\section{Figures}




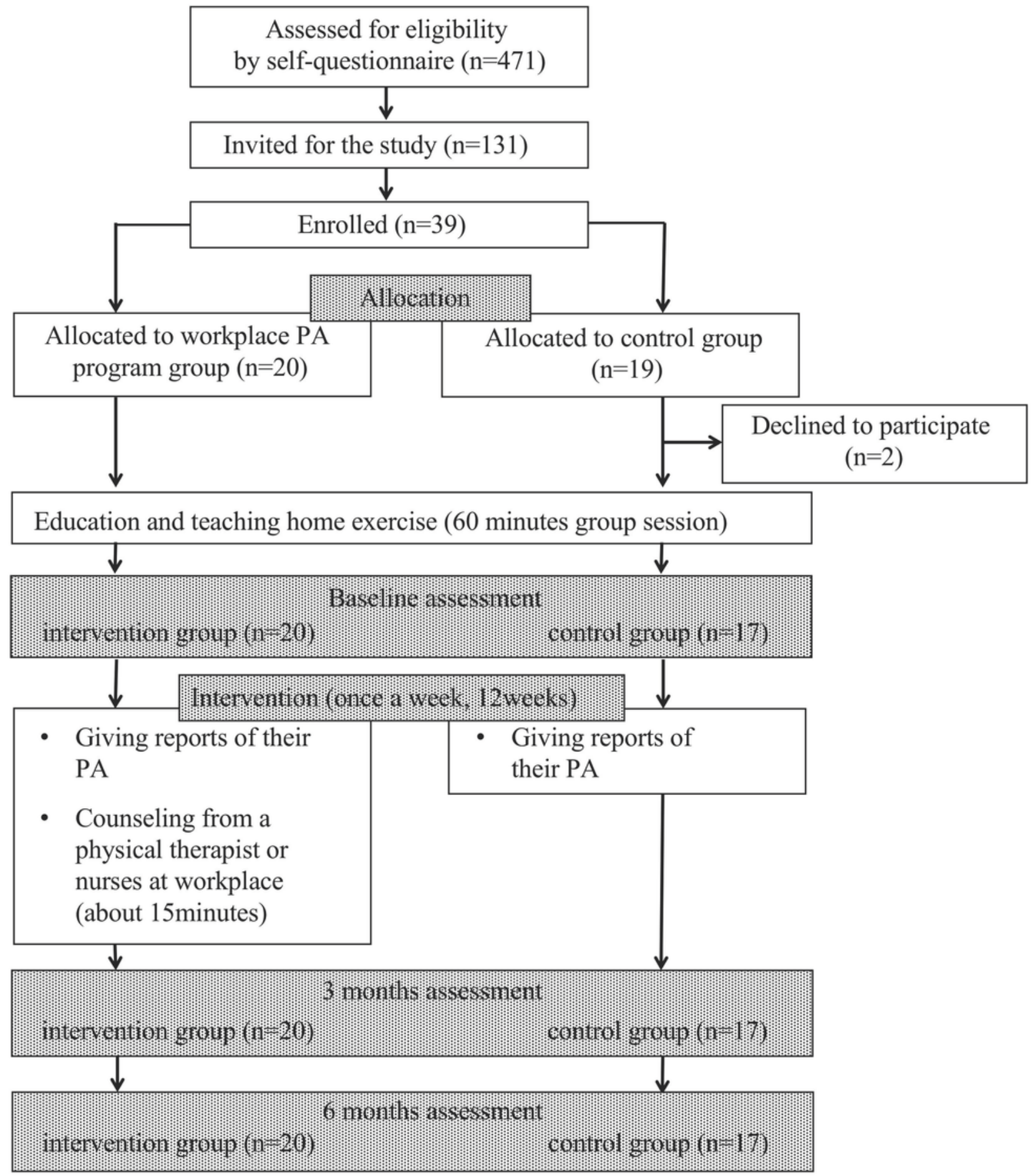

\section{Figure 1}

Trial profile

\section{Supplementary Files}

This is a list of supplementary files associated with this preprint. Click to download. 
- Table2.xlsx

- Table1.xlsx

- TIDieRChecklist.pdf

- Table3.xlsx

- CONSORT2010Checklist.pdf 